Pacific Journal of Mathematics

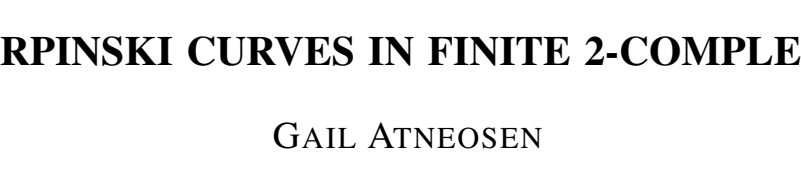




\section{SIERPINSKI CURVES IN FINITE 2-COMPLEXES}

\section{Gail H. AtNeosen}

In this note certain one-dimensional continua are defined for finite 2-complexes. These continua, called $S$-curves, are a generalization of the Sierpinski plane universal curve. By a 2-complex is meant a finite connected 2-dimensional euclidean polyhedron which has a triangulation such that every 1-simplex is the face of at least one 2-simplex. It is shown that any two $S$-curves in a 2-complex are homeomorphic. In addition, it is established that two 2-complexes (with the property that every 1-simplex in a triangulation is the face of two or more 2-simplexes) are homeomorphic if and only if the corresponding $S$-curves are homeomorphic.

In 1916 Sierpinski [4] described a one-dimensional continuum that is known as the Sierpinski plane universal curve. In 1958 Whyburn [7] defined the notion of an S-curve in a 2-sphere and established that an $S$-curve in a 2 -sphere is homeomorphic to the Sierpinski plane universal curve. In 1966 Borsuk [1] defined an $S$-curve in a surface. He established that any two $S$-curves in a given surface are homeomorphic and that two surfaces are homeomorphic if and only if the corresponding $S$-curves are homeomorphic. In this paper the same type of theorems are established for certain 2-complexes.

In order to define an S-curve in a 2-complex, it is necessary to introduce some terminology from Whittlesey [5] or [6]. A point $x$ in a 2-complex $K$ is a regular point if it has a neighborhood in $K$ homeomorphic to the plane (euclidean 2-dimensional space). The regular part of $K$ is the collection of all regular points in $K$. The points of $K$ which are not regular are called singular; the collection of all singular points in $K$ constitute the singular graph of $K$. Let $D_{1}, D_{2}, \cdots$ be a sequence of mutually disjoint closed dises contained in the regular part of $K$. Then $\mathrm{A}(K)=K-\bigcup_{i=1}^{\infty}$ Int $D_{i}$ (Int = interior in the sense of manifolds) is said to be an S-curve in $K$ provided that $\bigcup_{i=1}^{\infty} D_{i}$ is dense in $K$ and the diameters of the $D_{i}$ converge to zero. Note that if the 2-complex is also a surface, then this definition is precisely that of Borsuk [1, pp. 81-82].

LEMMA 1. Let $K$ be a 2-complex and $\Sigma$ an upper semi-continuous decomposition of $K$ with the property that every nondegenerate element of $\Sigma$ is contained in the regular part of $K$ and each nondegenerate element has arbitrarily small neighborhoods (in $K$ ) homeomorphic with the plane. Then the decomposition space $K_{\Sigma}$ is homeomorphic to $K$. 
Proof. It follows from results of Whittlesey [5, p. 843] that there exists a finite collection of bounded surfaces (compact, connected 2-manifolds with nonempty boundary) $M_{1}, \cdots, M_{j}$ such that $K$ is an identification space of their topological sum $M_{1}+\cdots+M_{j}$. The identification takes place on the boundaries of the surfaces. More precisely, if $f: M_{i}+\cdots+M_{j} \rightarrow K$ is the identification map, then $f$ restricted to the manifold interiors of the surfaces is a homeomorphism.

Consider the following diagram:

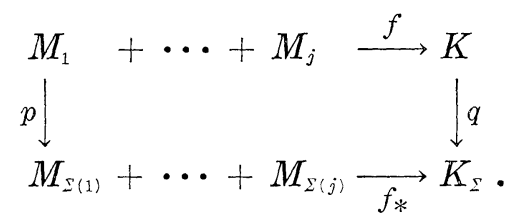

The upper semi-continuous decomposition $\Sigma$ of $K$ induces an upper semi-continuous decomposition $\Sigma(i)$ of $M_{i}, i=1, \cdots, j . \quad \Sigma(i)$ has as nondegenerate elements those sets $B$ such that $B=f^{-1}(b)$ where $b$ is a nondegenerate element of $\Sigma$. Let $p_{i}$ be the identification map of $M_{i}$ onto the decomposition space $M_{\Sigma(i)}, i=1, \cdots, j$. Let $p$ denote the identification map induced by the identification maps $p_{i}, i=1, \cdots, j$, and let $q$ denote the identification map for the decomposition $\Sigma$ of $K$. The map $f$ is a relation-preserving continuous map that is an identification. Hence, the induced map $f_{*}$ is continuous and is also an identification [2, Theorem 4.3, p. 126].

It follows from results of Borsuk [1, Theorem 3.1, p. 76] that $M_{i}$ is homeomorphic to $M_{\Sigma(i)}$. For each $i, i=1, \cdots, j$, the map $p_{i}$ restricted to $\mathrm{Bd} M_{i}(\mathrm{Bd}=$ boundary in the sense of manifolds $)$ is a homeomorphism onto $\mathrm{Bd} M_{\Sigma(i)}$. Furthermore, all the orientations of the boundaries are preserved by $p_{i}$, and so by [5, Lemma, p. 843] $p_{i}$ restricted to $\mathrm{Bd} M_{i}$ can be extended to a homeomorphism $h_{i}$ mapping $M_{i}$ onto $M_{\Sigma(i)}$.

Next consider the diagram:

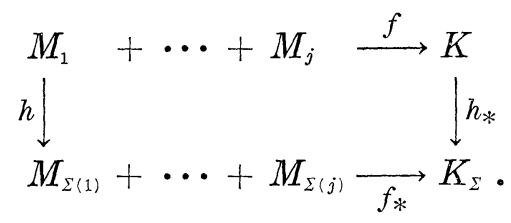

The homeomorphism $h$ is induced by the homeomorphisms $h_{i}, i=$ $1, \cdots, j$. As above, there exists a continuous mapping $h_{*}$ of $K$ onto $K_{\Sigma}$. Furthermore, $h_{*}$ is one-to-one. Since $K$ is compact and Hausdorff and $\Sigma$ is an upper semi-continuous decomposition, it follows from [3, Theorem 3-33, p. 133] that $K_{\Sigma}$ is Hausdorff. Thus $h_{*}$ is a one-toone continuous mapping of a compact space onto a Hausdorff space and hence is a homeomorphism. 
The proof of the next result closely parallels that of Borsuk [1, pp. 82-83] but is included for completeness.

THEOREM 1. Any two S-curves in a given 2-complex are homeomorphic.

Proof. Let $A=K-\bigcup_{i=1}^{\infty} \operatorname{Int} D_{i}$ be an $S$-curve in a 2-complex $K$. Consider the upper semi-continuous decomposition $\Sigma$ of $K$ whose nondegenerate elements are the discs $D_{i}$. $K$ is homeomorphic to $K_{\Sigma}$ by Lemma 1 . The subset of the decomposition space $K_{\Sigma}$ consisting of points $d_{i}$ corresponding to the discs $D_{i}$ is countable and is contained in the regular part of $K_{\Sigma}$. If $K$ has triangulation $T_{0}$, there exists a "curved" triangulation $T$ of $K_{\Sigma}$ isomorphic to $T_{0}$ such that no point $d_{i}, i=1,2, \cdots$, belongs to the 1-dimensional skeleton $Z$ of $T$. The skeleton $Z$ may be considered as lying in the set $K-\bigcup_{i=1}^{\infty} D_{i}$. Thus a triangulation $T$ of $K$ is obtained that is isomorphic to $T_{0}$ with the property that every disc $D_{i}$ lies in the interior of a 2-simplex of $K$.

Similarly, if $A^{\prime}=K-\bigcup_{i=1}^{\infty}$ Int $D_{i}^{\prime}$ is another $S$-curve in $K$, it follows from the above argument that there exists another triangulation $T^{\prime}$ of $K$ isomorphic to $T$ such that every disc $D_{i}$ lies in the interior of a 2 -simplex of $T^{\prime}$. Let $Z^{\prime}$ denote the 1-skeleton.

Since $T$ and $T^{\prime}$ are isomorphic, there is a homeomorphism $h$ mapping $K$ onto $K$ such that each 2-simplex $E$ of $T$ is mapped by $h$ onto a 2-simplex $E^{\prime}$ of $T^{\prime}$. Then $E \cap A$ and $E^{\prime} \cap A^{\prime}$ may be viewed as $S$-curves in a 2-sphere, and $h$ as a homeomorphism mapping the outer boundary of $E \cap A$ onto the outer boundary of $E^{\prime} \cap A^{\prime}$. Thus by a result of Whyburn [7, p. 322], $h$ restricted to $\mathrm{Bd} E$ can be extended to a homeomorphism $h_{E}$ mapping $E \cap A$ onto $E^{\prime} \cap A^{\prime}$. The mapping $h$ can then be extended to a homeomorphism mapping $A$ onto $A^{\prime}$ by defining $h(x)=h_{E}(x)$ for $x \in A$ and $x$ contained in the 2-simplex $E$ of $T$.

Next it is established that certain 2-complexes are completely characterized by their $S$-curves. Let $K$ be the union of all the proper faces of a 3-simplex and let $K^{\prime}$ be a 2 -simplex. Then $A(K)$ is homeomorphic to $A\left(K^{\prime}\right)$ but $K$ is not homeomorphic to $K^{\prime}$. This example shows that extra conditions are needed on the 2-complexes for such a characterization. The sufficient conditions are stated in Theorem 2.

First, some terminology from Borsuk [1, p. 84] must be introduced. Let $A(K)=K-\bigcup_{i=1}^{\infty}$ Int $D_{i}$ be an $S$-curve associated with a 2-complex $K$. The set $\mathrm{Bd} A(K)=\bigcup_{i=1}^{\infty} \mathrm{Bd} D_{i}$ is said to be the boundary of $A(K)$. The set Int $A(K)=A(K)-\mathrm{Bd} A(K)$ is said to be the interior of $A(K)$. Singular interior points of $A(K)$ are those interior points contained in the singular graph of $K$.

Let $S$ be a Sierpinski plane universal curve and $I$ an arc (a space homeomorphic to the closed interval $[0,1])$. Let $Y$ denote the space 
obtained by identifying an endpoint of $I$ with an interior point $x$ of $S$. Observe that every interior point of $S$ is interior to arbitrarily small rectangular plane neighborhoods whose boundaries lie in $S$. Hence $Y$ is not embeddable in the plane. This fact will be used in the proof of the following lemma.

LEMMA 2. Let $K$ be a 2-complex such that every 1-simplex is the face of two or more 2-simplexes, and let $A(K)$ be the associated $S$-curve. $A$ point $x$ in $A(K)$ is a singular interior point if and only if no neighborhood of $x$ in $A(K)$ is embeddable in the plane.

Proof. It is clear that if $x$ does not have a neighborhood in $A(K)$ embeddable in the plane, then $x$ does not have such a neighborhood in $K$. Thus $x$ belongs to the singular graph of $K$ and is a singular interior point of $A(K)$.

Conversely, suppose $x$ is a singular interior point. Then $x$ is an element of the singular graph of $K$. To show that no neighborhood of $x$ in $A(K)$ is embeddable in the plane it suffices to establish that every neighborhood of $x$ in $A(K)$ contains a subset homeomorphic to $Y$ (as defined above). Whittlesey has classified the singular points of a 2-complex. His definitions [5, p. 842] are used to consider the various cases.

Case 1. $x$ is a line singularity. Then $x$ has arbitrarily small neighborhoods in $K$ homeomorphic to the space obtained by identifying the $x$-axes of $n$ ( $n \geqq 3$ by the hypothesis of the lemma) copies of the closed euclidean half-plane $y \geqq 0$. It follows that every neighborhood of $x$ in $A(K)$ contains a subset homeomorphic to $Y$.

Case 2. $x$ is a conical point. Then $x$ has arbitrarily small neighborhoods in $K$ homeomorphic to the set which is obtained if $n$ copies $(n \geqq 2)$ of the plane are identified at the origin. Again every neighborhood of $x$ in $A(K)$ contains a copy of $Y$.

Case 3. $x$ is a node. A node is necessarily a vertex in any triangulation of $K$. Let $T$ be a triangulation of $K$. Then the regular part of the Star of $x$ falls into components each of which is a cone with $x$ at the vertex or is, topologically, an open triangle with $x$ as a vertex and with two singular edges, both edges having $x$ as a vertex, and the edges may be distinct or coincide. Since by hypothesis every 1-simplex is the face of two or more 2 -simplexes, every neighborhood in $A(K)$ of a node will contain a copy of $Y$.

All possible singular interior points have been considered and the proof is completed. 
THEOREM 2. Let $K$ and $K^{\prime}$ be 2-complexes such that every 1-simlex in a triangulation of $K$ or $K^{\prime}$ is the face of two or more 2-simplexes. Let $A(K)$ and $A\left(K^{\prime}\right)$ be the S-curves associated with $K$ and $K^{\prime}$ respectively. Then $A(K)$ is homeomorphic to $A\left(K^{\prime}\right)$ if and only if $K$ is homeomorphic to $K^{\prime}$.

Proof. Let $h$ mapping $A(K)$ onto $A\left(K^{\prime}\right)$ be a homeomorphism. Let Int $D_{i}$ be an open disc in $K-A(K)$ with $\mathrm{Bd} D_{i}=C$ which is contained in $A(K)$. Consider $h(C)=C^{\prime}$. Then $C^{\prime}$ is a simple closed curve in $A\left(K^{\prime}\right)$. Next it is established that $C^{\prime}=\mathrm{Bd} D_{i}^{\prime}$ where $A\left(K^{\prime}\right)=$ $K^{\prime}-\bigcup_{i=1}^{\infty} \operatorname{Int} D_{i}^{\prime}$.

If $x \in C$, then there exists a neighborhood of $x$ in $A(K)$ embeddable in the plane. By Lemma $2, h(x)$ is not a singular interior point of $A\left(K^{\prime}\right)$. Furthermore, if $x \in C$ then $x$ is contained in the interior of an arc in $C$ that does not locally decompose $A(K)$. It follows from $[1, \mathrm{p} .84]$ that $C^{\prime}$ is contained in $\mathrm{Bd} A\left(K^{\prime}\right)$. Hence $C^{\prime}=\mathrm{Bd} D_{i}^{\prime}$ for some $i$. For each $i$ the map $h$ restricted to the $\mathrm{Bd} D_{i}$ can be extended to a homeomorphism $h_{i}$ mapping the disc $D_{i}$ onto the disc $D_{i}^{\prime}$. Since the diameters of the sets $h\left(\mathrm{Bd} D_{i}\right)$ converge to zero, the diameters of the dises $D_{i}^{\prime}$ converge to zero. Extend $h$ to a mapping $h^{\prime}$ of $K$ onto a subset of $K^{\prime}$ by defining $h^{\prime}(x)=h(x)$ for $x$ in $A(K)$ and $h^{\prime}(x)=h_{i}(x)$ for $x$ in Int $D_{i}$. Then $h^{\prime}$ is a mapping of $K$ onto a subset of $K^{\prime}$. But since $h(A(K))=A\left(K^{\prime}\right), h^{\prime}$ is also onto $K^{\prime}$; and $K$ is homeomorphic to $K^{\prime}$.

The converse follows from Theorem 1.

The reader will able to make the necessary modifications to extend these results by himself to arbitrary finite 2-complexes.

\section{REFERENCES}

1. K. Borsuk, On embedding curves in surface, Fund. Math., 59 (1966), 73-89.

2. J. Dugundji, Topology, Allyn and Bacon, Inc., (1966).

3. J. G. Hocking and G. S. Young, Topology, Addison-Wesley, (1961).

4. W. Sierpinski, Sur une courbe cantorienne qui contient une image biunivoque et continue de toute courbe donée, Comptes Rendus Acad. Sci., Paris, 162 (1916), 629-632.

5. F. F. Whittlesey, Classification of finite 2-complexes, Proc. Amer. Math. Soc., 9 (1958), 841-845.

6. - Finite surfaces $I, I I$, local structure and canonical form, Math. Mag., 34 (1960), no. 1 11-22, no. 2 67-68.

7. G. T. Whyburn, Topological characterization of the Sierpinski curve, Fund. Math., 45 (1958), 320-324.

Received June 12, 1972 and in revised form October 16, 1972. 



\section{PACIFIC JOURNAL OF MATHEMATICS}

\section{EDITORS}

RICHARD ARENS (Managing Editor) University of California

Los Angeles, California 90024

R. A. BeaUmont

University of Washington Seattle, Washington 98105
J. DUGUNDJI*

Department of Mathematics University of Southern California Los Angeles, California 90007

D. Gilbarg and J. Milgram Stanford University

Stanford, California 94305

\section{ASSOCIATE EDITORS}

E. F. BECKENBACH

B. H. NeUmanN

F. WOLF

K. YoSHIDA

\section{SUPPORTING INSTITUTIONS}

\section{UNIVERSITY OF BRITISH COLUMBIA CALIFORNIA INSTITUTE OF TECHNOLOGY UNIVERSITY OF CA.LIFORNIA MONTANA STATE UNIVERSITY UNIVERSITY OF NEVADA NEW MEXICO STATE UNIVERSITY OREGON STATE UNIVERSITY UNIVERSITY OF OREGON OSAKA UNIVERSITY}

UNIVERSITY OF SOUTHERN CALIFORNIA STANFORD UNIVERSITY UNIVERSITY OF TOKYO UNIVERSITY OF UTAH WASHINGTON STATE UNIVERSITY UNIVERSITY OF WASHINGTON AMERICAN MATHEMATICAL SOCIETY NAVAL WEAPONS CENTER

* C. R. DePrima California Institute of Technology, Pasadena, CA 91109, will replace J. Dugundji until August 1974. 


\section{Pacific Journal of Mathematics}

\section{Vol. 50, No. $1 \quad$ September, 1974}

Gail Atneosen, Sierpinski curves in finite 2-complexes.............. 1

Bruce Alan Barnes, Representations of $B^{*}$-algebras on Banach spaces .... 7

George Benke, On the hypergroup structure of central $\Lambda(p)$ sets ....... 19

Carlos R. Borges, Absolute extensor spaces: a correction and an

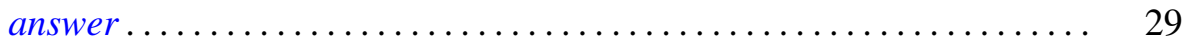

Tim G. Brook, Local limits and tripleability .................. 31

Philip Throop Church and James Timourian, Real analytic open maps .... 37

Timothy V. Fossum, The center of a simple algebra ............... 43

Richard Freiman, Homeomorphisms of long circles without periodic

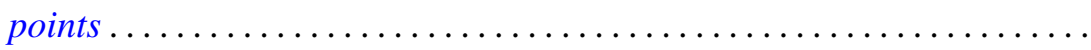

B. E. Fullbright, Intersectional properties of certain families of compact

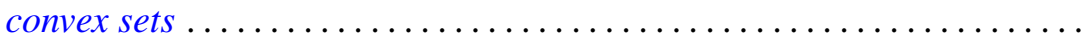

Harvey Charles Greenwald, Lipschitz spaces on the surface of the unit

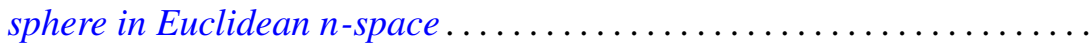

Herbert Paul Halpern, Open projections and Borel structures for

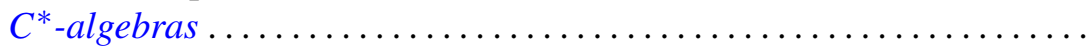

Frederic Timothy Howard, The numer of multinomial coefficients divisible

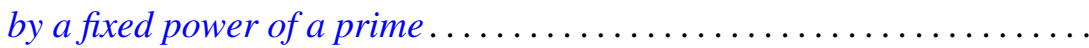

Lawrence Stanislaus Husch, Jr. and Ping-Fun Lam, Homeomorphisms of manifolds with zero-dimensional sets of nonwandering points........ 109

Joseph Edmund Kist, Two characterizations of commutative Baer rings ...

Lynn McLinden, An extension of Fenchel's duality theorem to saddle functions and dual minimax problems ................

Leo Sario and Cecilia Wang, Counterexamples in the biharmonic classification of Riemannian 2-manifolds...

Saharon Shelah, The Hanf number of omitting complete types ...

Richard Staum, The algebra of bounded continuous functions into a

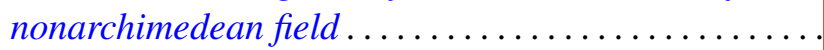

James DeWitt Stein, Some aspects of automatic continuity ..

Tommy Kay Teague, On the Engel margin

John Griggs Thompson, Nonsolvable finite groups all of whose local subgroups are solvable, $V \ldots \ldots \ldots \ldots \ldots \ldots \ldots \ldots$

Kung-Wei Yang, Isomorphisms of group extensions 